\title{
Bayesian Motion Recovery Framework for Myocardial Phase-Contrast Velocity MRI
}

\author{
Andrew Huntbatch, Su-Lin Lee, David Firmin, and Guang-Zhong Yang \\ Royal Society/Wolfson Foundation MIC Laboaratory, Imperial College London \\ \{a.huntbatch, su-lin.lee,d.firmin,g.z.yang\}@imperial.ac.uk
}

\begin{abstract}
Detailed assessment of myocardial motion provides a key indicator of ventricular function, enabling the early detection and assessment of a range of cardiac abnormalities. Existing techniques for myocardial contractility analysis are complicated by a combination of factors including resolution, acquisition time, and consistency of quantification results. Phase-contrast velocity MRI is a technique that provides instantaneous, in vivo measurement of tissue velocity on a per-voxel basis. It allows for the direct derivation of contractile indices with minimal post-processing. For this method to be clinically useful, SNR and image artifacts need to be addressed. The purpose of this paper is to present a Maximum a posteriori (MAP) restoration technique for high quality myocardial motion recovery. It employs an accurate noise modeling scheme and a generalized Gaussian Markov random field prior tailored for the myocardial morphology. The quality of the proposed method is evaluated with both simulated myocardial velocity data with known ground truth and in vivo phase-contrast MR velocity acquisitions from a group of normal subjects.
\end{abstract}

\section{Introduction}

The early detection and accurate assessment of abnormal myocardial contractility is important to the diagnosis and treatment of a range of cardiac diseases. Historically, Magnetic Resonance (MR) tagging has been widely used for assessing intrinsic cardiac motion but issues related to resolution and complex post-processing involved remain major obstacles for its routine clinical use [1]. The recent development in harmonic phase (HARP) imaging, which exploits the phase properties of MR tagging, has significantly extended its clinical value, particularly for rapid assessment of stress induced contractility changes [2]. DENSE imaging has also proven to be useful in the measurement of strain through the tracking of tissue over time. However, for the direct measurement of contractility, phase-contrast velocity imaging is able to instantaneously measure myocardial velocity on a per-voxel basis. This offers the potential of more detailed analysis of intramural myocardial contractility, with levels of detail beyond other existing techniques. However, the consistency of myocardial velocity mapping still remains a major problem, and there are a number of factors that can influence the SNR that is achievable for patient studies. This is due to the relatively low velocity magnitude of the myocardial motion and artifacts arising from the ventricular flow patterns. The recent use of black-blood saturation sequences has improved the overall quality of the myocardial velocity data, but for accurate biomechanical quantification or modeling, phase velocity restoration is essential. 
Hitherto, the Total Variation (TV) approach to image restoration has already received considerable attention across a wide range of fields. The technique was originally proposed by Rudin et al. [3] and involves the use of the TV norm, an $\mathrm{L}_{1}$ norm of the derivative, rather than the more traditional use of least-squares methods based on $\mathrm{L}_{2}$. While the use of $\mathrm{L}_{1}$ methods had been proposed before, their non-linear nature and high complexity in comparison to least-squares estimates had prevented the development of effective and robust image restoration algorithms. A constrained minimization algorithm has been used to smooth images by reducing the total variation of the image, subject to the statistical constraint that the variation of the removed noise component matched a priori knowledge.

An extension of TV restoration to vector-valued images was made by Blomgren et al. [4] in their paper on color image restoration. Subsequently, this approach to TV restoration was applied to MR velocity data [5] and extended through the use of the first order Lagrangian technique to derive an optimal solution to the constrained minimization problem. A significant observation of this work was that the restoration algorithm was not greatly affected by the estimated noise variance, thus significantly enhancing the practical usability of the technique as reliable noise variance estimation is difficult in practice. Smooth boundary-preserving velocity fields could be produced using a wide range of noise estimates.

In parallel, restorative algorithms based on Bayes theorem have also been applied to a range of fields, allowing a great degree of flexibility in terms of modeling both the noise and underlying image structures. In particular, the maximum a posteriori (MAP) approach allows prior knowledge of image data to be readily combined with knowledge of the noise and distortion introduced during the imaging process. In medical image computing, a number of MAP based techniques have been developed [6] and the principles employed have also been used for super-resolution image construction [7].

The purpose of this paper is to introduce a MAP based velocity restoration scheme with accurate identification of the noise distribution and image prior based on a generalized Markov Random Field (MRF). The method developed is validated with in vivo data collected from a group of 7 normal subjects.

\section{Method}

For MAP based techniques, the correct choice of image prior is essential. To this end, MRFs have attracted considerable attention due to their inherent flexibility and consideration of local image context which permits efficient computation of the MAP estimate. The central component of a MRF is a potential function which assigns a probability to the configuration of a pixel in relation to its neighbors. The choice of the exact nature of this function is an important step in the successful application of the prior model. To this end, generalized Gaussian MRFs provide a flexible yet powerful approach to the image prior [8], with the benefit of scale invariance. It is worth noting that another prior model that can be used is the Huber prior [9], which is especially suited to the preservation of sharp edges due to its transition from a quadratic to linear function at larger image variations. 


\subsection{MAP Based Velocity Restoration}

Velocity images acquired through the phase-contrast sequence are corrupted by additive noise resulting from noise generated in the receiving coil and other sources. Therefore, the observed velocity image $y$ can be considered as the sum of the underlying clean image $x$ that we wish to determine and some additive noise image. The MAP estimate of the clean image can be formulated through Bayes theorem by taking into account both prior knowledge of the underlying tissue and the probability distribution of the noise component, i.e.,

$$
\begin{aligned}
\hat{x} & =\underset{x}{\arg \max }[p(x \mid y)] \\
& =\underset{x}{\arg \max }[\log p(y \mid x)+\log p(x)]
\end{aligned}
$$

where $p(x \mid y)$ is the likelihood function and $p(x)$ is the image prior. The maximization of this $\log$ probability yields the restored image.

Phase-contrast velocity imaging employs a bi-polar gradient to encode the velocity of the tissue in the phase of the complex image. The various stages of image acquisition introduce different noise components. However, after complex difference processing, it can be assumed that both the complex and real values of the velocityencoded image are corrupted by additive Gaussian noise [10]. However, since phase angle is obtained through the non-linear arctangent function of the ratio of the real and complex images values, the probability distribution function (p.d.f) for phasenoise $(\Delta \theta)$ has the following form [11]:

$$
\begin{gathered}
p_{\Delta \theta}(\Delta \theta)=\frac{1}{2 \pi} e^{-A^{2 / 2 \sigma^{2}}}\left[1+\frac{A}{\sigma} \sqrt{2 \pi} \cos \Delta \theta e^{A^{2} \cos ^{2} \Delta \theta / 2 \sigma^{2}}\right. \\
\left.\cdot \frac{1}{\sqrt{2 \pi}} \int_{-\infty}^{\frac{A \cos \Delta \theta}{\sigma}} e^{-\frac{x^{2}}{2}} d x\right]
\end{gathered}
$$

where $(\Delta \theta)$ is the change in observed phase, $\sigma^{2}$ is the variance of the complex and real Gaussian noise components, and $A$ is the amplitude of the MR signal. As has previously been reported [11], the above distribution reduces to far less complex expressions in the cases where SNR is zero and SNR is high, representing uniform and Gaussian distributions respectively. However, in many situations where it is desirable to apply phase-contrast imaging, particularly for the imaging of low-velocity tissue such as the myocardium, SNR is low and the suitability of the Gaussian approximation cannot be guaranteed.

With regard to the image prior, generalized Gaussian MRFs provide a good model but it requires careful selection of the appropriate parameters. The general form of the logarithmic generalized Gaussian MRF prior can be represented as:

$$
\log p(x)=-\frac{1}{2 \sigma^{2}} \sum_{\{i, j\} \in C} g_{i, j}\left|x_{i}-x_{j}\right|^{p}
$$

where $\sigma^{2}$ is the scale parameter of the Gaussian function, $g$ is a neighborhood weighting kernel, $C$ is the set of all cliques within the image, and $p$ is a parameter varying 
between 1 and 2. The optimal value of $\sigma^{2}$ can be found using a maximum likelihood (ML) approach based on the observed noisy data [12]. The parameter $p$ affects the penalization of edges in the image and varies the model between a linear one at $p=1$ and a Gaussian at $p=2$. In the work by Rey [13], the value of $p$ was investigated with the value of 1.2 used in this paper suggested as the best compromise between edge preservation and noise suppression.

For 2D image restoration, the selection of the pixel neighborhood is usually straightforward. However, the morphology of the myocardium is such that a neighborhood shape function aligned with the cylindrical polar coordinate system is more appropriate than Cartesian coordinates. For this study, the neighborhood selection considers the radial and circumferential location on each neighboring slice to ensure the correct contextual relationship is maintained.

By combining the generalized Gaussian MRF with the log likelihood function (2) gives, the following minimization function can be derived:

$$
\hat{x}=\arg \min _{x}\left\{-\sum_{s \in S} \log p_{\Delta \theta}\left(x_{s}-y_{s}\right)+\frac{1}{2 \sigma_{x}^{2}} \sum_{s \in S} \sum_{j \in N s} g_{i, j}\left|x_{i}-x_{j}\right|^{p}\right\}
$$

where $\sigma_{x}$ is the scale parameter for the generalized Gaussian MRF. An important feature of the components of this function is convexity, which enables the global minimum to be found in all cases. In general, the choice of the minimization technique is not of great significance but in this paper a Broyden-Fletcher-GoldfarbShannon (BFGS) algorithm is used to derive the MAP estimate.

\subsection{Data Acquisition}

The myocardial velocity data used for this study was acquired from a $1.5 \mathrm{~T}$ Siemens Sonata MRI scanner. A three-directional velocity mapping sequence $(\mathrm{TR}=3 \mathrm{~ms}$, $\mathrm{TE}=7.1 \mathrm{~ms}$ ) was used to image the left ventricle myocardium of 7 normal subjects with informed consent. To avoid respiratory induced cardiac motion, a gating protocol using a MR diaphragmatic navigator echo was used to remove motion artifacts. The velocity sequence is of the gradient-echo type with an in-plane voxel resolution of $1.17 \times 1.17 \mathrm{~mm}$, FOV of $300 \times 300 \mathrm{~mm}$, and a velocity encoding range of $\pm 150 \mathrm{mms}^{-1}$. A spatio-temporal unwrapping algorithm [14] was used to correct for the small amount of wrapping that occurred, which also at the same time extended the effective dynamic range of the velocity sequence.

During the experiment, velocity images were acquired over a series of short-axis slices positioned at $8 \mathrm{~mm}$ intervals with $8 \mathrm{~mm}$ thickness from the apex to the base of the heart. At each slice, the three orthogonal velocity components, as well as the reference image without velocity encoding, were obtained. In addition, two long-axis intensity images were acquired to facilitate the delineation of the valve plane of the left ventricle throughout different phases of the cardiac cycle. Approximately 12 slice positions and 15 time frames were generated for each patient. The 3D velocity fields for each slice were generated by combining the 3 phase-contrast images at each slice.

\subsection{Numerically Simulated Dataset}

To assess the quality of the restoration with known ground truth, a simulated data set was generated using a simplified mechanical model of the left ventricle. This dataset 
used a hexahedral solid mesh that is morphologically similar to the LV from the apex to the valve plane with a cyclic shortening, torsion and contractile motion. The incompressibility of myocardial tissue was incorporated into the model by ensuring that the divergence of the velocity field was zero at all points. From this model, a set of short axis slices were generated at fixed positions at each time frame. Gaussian noise with variance $(\sigma=0.3)$ was added to each slice by deriving underlying real and complex images for each velocity component.

\subsection{Myocardial Contractility Assessment}

To facilitate inter-subject comparison, a shape-based interpolation technique was used to generate 3D distributions of the velocity data. First, the epicardial and endocardial borders were manually defined and, along with the valve plane, regular samples were acquired within a cylindrical polar coordinate system using cubic spline interpolation over the entire myocardium. The cylindrical polar coordinate system is ideally suited to the study of LV dynamics as each axis corresponds to a basic myocardial motion: contraction, torsion and shortening. The myocardium was divided into 48 segments longitudinally, 64 circumferentially and 4 radially to give a hexahedral mesh. These element quantities were chosen to give similar resolution along each axis and to closely match the voxel size of the underlying images. Strain-rate was calculated as the spatial derivative of velocity and the tensor formed by the mixture of the different directional components provides useful indices such as magnitude of tissue contractility and the principal directions of contraction. They were calculated at each point on the velocity map to demonstrate the quality of the restoration. The eigenvectors of the strain-rate tensor demonstrate the principal directions of strain-rate within the tissue and are visualized using ellipsoids.

\section{Results and Discussion}

Fig. 1 illustrates an area of the myocardium from the mid-lateral region of the simulated data at mid-diastole, demonstrating the effect of noise on the resulting strainrates calculation and the improvement achieved due to the proposed MAP restoration scheme. It can be seen that the noise introduces areas of apparent high strain-rate and that these are significantly reduced by the proposed MAP restoration algorithm.

Fig. 2 illustrates the mean, restored radial, circumferential, and longitudinal velocity components for all the subjects studied. This forms a velocity atlas (albeit being limited to only 7 subjects studied), which can be used as a reference for patient studies.

To further illustrate the quality improvement achieved, Fig. 3 shows the distribution of strain-rates for one of the subjects studied. The results are represented over 16 segments of the standard AHA 17-segment model [15], but with the apical segment omitted. The derived distribution of the radial and longitudinal strain-rates follows the expected contractile pattern for a normal subject, demonstrating radial contraction, longitudinal 


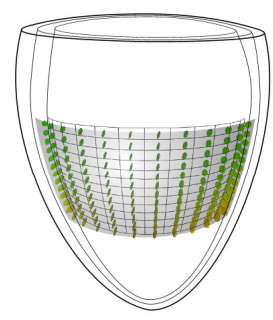

(a)
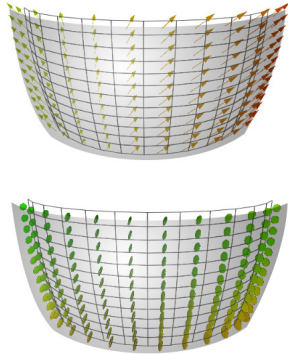

(b)
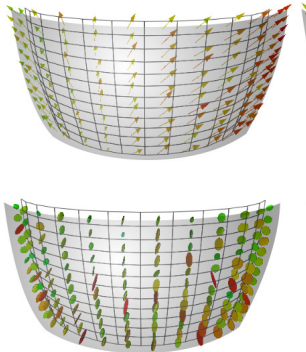

(c)
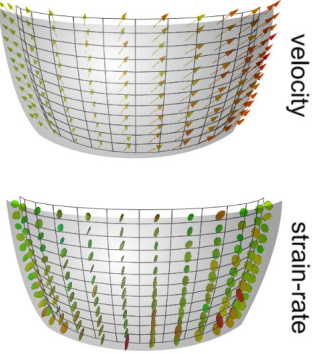

(d)

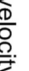

Fig. 1. Effect of restoration on a mid-lateral section of a numerically simulated LV, showing (a) the location of the selected LV region, (b) the original ground truth data, (c) data contaminated with noise, and (d) the restored velocity distribution and its corresponding strain rate

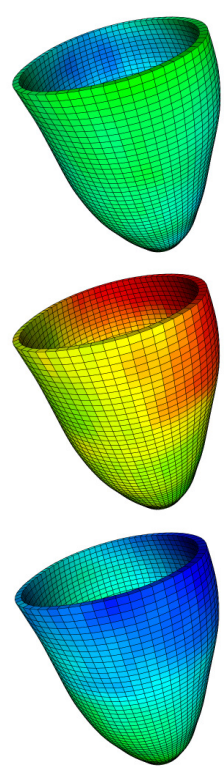

(a)
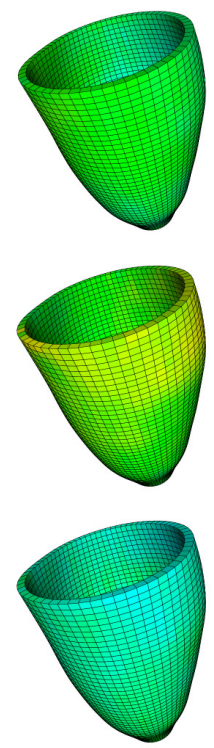

(b)
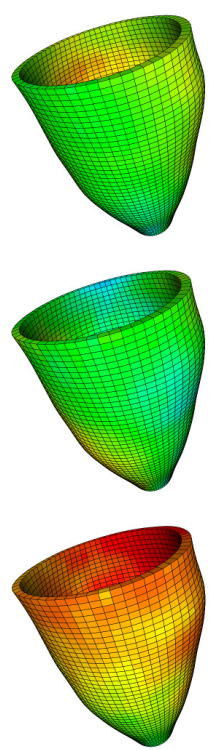

(c)

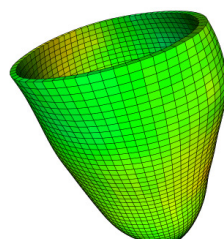

$+140 \mathrm{mms}^{-1}$
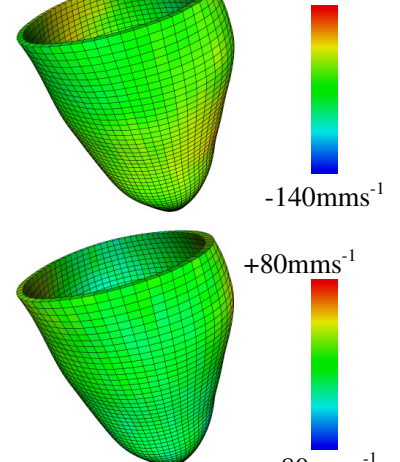

$+80 \mathrm{mms}^{-1}$
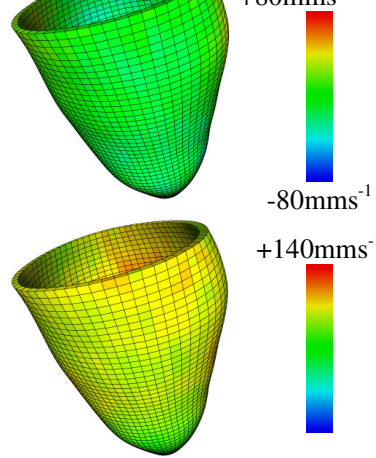

(d)

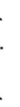

(1)



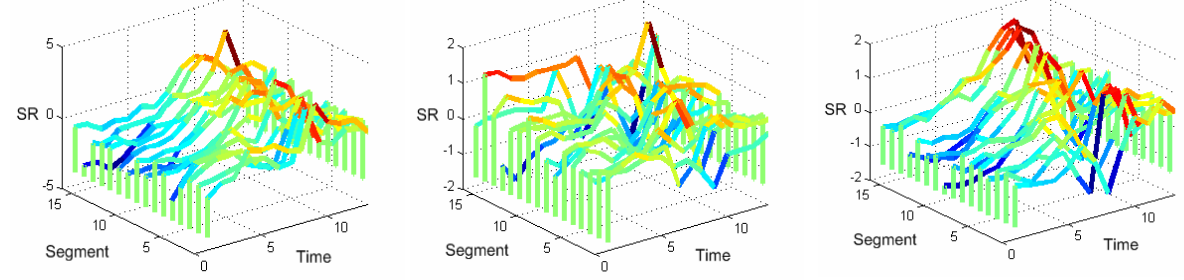

Fig. 3. The radial, circumferential and longitudinal strain-rates (from left to right) for one of the subjects studied across the 16 myocardial segments

Table 1. The mean and variance of mid-systolic radial, circumferential and longitudinal components of the derived strain-rates over all 16 segments of the subjects studied

\begin{tabular}{crrrrrrrr}
\hline \hline Segment & 1 & 2 & 3 & 4 & 5 & 6 & 7 & 8 \\
\hline $\mathrm{r}$ & $\mathbf{- 1 . 3 1}$ & $\mathbf{- 1 . 5 5}$ & $\mathbf{- 1 . 7 3}$ & $\mathbf{- 1 . 1 4}$ & $\mathbf{- 1 . 4 5}$ & $\mathbf{- 1 . 2 5}$ & $\mathbf{- 1 . 1 9}$ & $\mathbf{- 1 . 4 4}$ \\
& 0.40 & 0.66 & 0.71 & 0.30 & 1.04 & 0.29 & 0.42 & 0.44 \\
\hline$\theta$ & $\mathbf{0 . 3 2}$ & $\mathbf{0 . 1 9}$ & $\mathbf{0 . 0 0}$ & $\mathbf{- 0 . 5 0}$ & $\mathbf{- 0 . 1 3}$ & $\mathbf{0 . 1 0}$ & $\mathbf{- 0 . 0 8}$ & $\mathbf{0 . 0 4}$ \\
& 0.22 & 0.15 & 0.13 & 0.25 & 0.32 & 0.17 & 0.21 & 0.52 \\
\hline 1 & $\mathbf{0 . 5 7}$ & $\mathbf{- 0 . 5 3}$ & $\mathbf{- 0 . 8 5}$ & $\mathbf{- 0 . 8 9}$ & $\mathbf{- 0 . 4 4}$ & $\mathbf{- 0 . 5 5}$ & $\mathbf{- 0 . 3 7}$ & $\mathbf{- 0 . 6 3}$ \\
& 0.12 & 0.26 & 0.48 & 0.22 & 0.41 & 0.23 & 0.10 & 0.31 \\
\hline & 9 & 10 & 11 & 12 & 13 & 14 & 15 & 16 \\
\hline $\mathrm{r}$ & $\mathbf{- 1 . 4 4}$ & $\mathbf{- 2 . 0 7}$ & $\mathbf{- 1 . 7 6}$ & $\mathbf{- 1 . 5 1}$ & $\mathbf{- 0 . 9 3}$ & $\mathbf{- 1 . 7 0}$ & $\mathbf{- 2 . 3 8}$ & $\mathbf{- 0 . 8 5}$ \\
& 0.65 & 0.48 & 0.42 & 0.27 & 0.53 & 0.27 & 0.64 & 0.28 \\
\hline$\theta$ & $\mathbf{0 . 2 5}$ & $\mathbf{0 . 1 8}$ & $\mathbf{- 0 . 1 2}$ & $\mathbf{- 0 . 2 7}$ & $\mathbf{- 0 . 8 0}$ & $\mathbf{1 . 1 4}$ & $\mathbf{0 . 6 1}$ & $\mathbf{- 0 . 9 7}$ \\
& 0.25 & 0.35 & 0.18 & 0.36 & 0.68 & 0.85 & 0.48 & 0.89 \\
\hline 1 & $\mathbf{- 0 . 6 6}$ & $\mathbf{- 0 . 5 3}$ & $\mathbf{- 0 . 8 7}$ & $\mathbf{- 0 . 6 3}$ & $\mathbf{- 0 . 7 9}$ & $\mathbf{- 0 . 3 7}$ & $\mathbf{- 0 . 8 3}$ & $\mathbf{- 0 . 7 1}$ \\
& 0.63 & 0.26 & 0.34 & 0.20 & 0.28 & 0.24 & 0.23 & 0.23 \\
\hline \hline
\end{tabular}

\section{Conclusion}

In this paper, we have presented a MAP-based technique for the restoration of myocardial motion fields acquired through phase-contrast MRI and demonstrated the extraction of high-resolution contractility metrics. Myocardial velocity imaging has the potential to accurately determine the low-level functioning of the myocardium with a view to improved detection and assessment of myocardial disease. Because of the relatively low SNR of the phase-contrast sequence, the use of robust restoration techniques is crucial to the subsequent analysis of derived biomechanical indices. The technique we have developed in this work has been shown as highly successful in the creation of high-resolution strain-rate maps of the myocardium as demonstrated by results from a group of normal subjects. This facilitates the future adoption of phase-contrast velocity imaging in routine clinical environments. 


\section{References}

1. Masood, S., Gao, J., Yang, G.Z.: Virtual tagging: numerical considerations and phantom validation. IEEE Transactions on Medical Imaging 21, 1123-1131 (2002)

2. Osman, N.F., Kerwin, W.S., McVeigh, E.R., Prince, J.L.: Cardiac motion tracking using CINE harmonic phase (HARP) magnetic resonance imaging. Magnetic resonance in Medicine 42, 1048-1060 (1999)

3. Rudin, L., Osher, S., Emad, F.: Nonlinear total variation based noise removal algorithms. Physica D 60, 259-268 (1992)

4. Blomgren, P., Chan, T.F.: Color TV: total variation methods for restoration of vectorvalued images. IEEE Transactions on Image Processing 7, 304-309 (1998)

5. Ng, Y.H.P., Carmo, B.S., Yang, G.Z.: Flow Field Abstraction and Vortex Detection for MR Velocity Mapping. Medical Image Computing and Computer-Assisted Intervention (2003)

6. Basu, S., Fletcher, T., Whitaker, R.: Rician noise removal in diffusion tensor MRI. Medical Image Computing and Computer Assisted Intervention 9, 117-125 (2006)

7. Pickup, L.C., Capel, D.P., Roberts, S.J., Zisserman, A.: Bayesian Methods for Image Super-Resolution (2007)

8. Bouman, C., Sauer, K.: A generalized Gaussian image model for edge-preserving MAP estimation. IEEE Transactions on Image Processing 2, 296-310 (1993)

9. Huber, P.: Robust Statistics. John Wiley \& Sons, New York (1981)

10. Andersen, A.H., Kirsch, J.E.: Analysis of noise in phase contrast MR imaging. Medical Physics 23, 857-869 (1996)

11. Gudbjartsson, H., Patz, S.: The rician distribution of noisy mri data. Magnetic Resonance in Medicine 34, 910-914 (1995)

12. Saquib, S.S., Bouman, C.A., Sauer, K.: ML parameter estimation for Markov random fields with applications to Bayesian tomography. IEEE Transactions on Image Processing 7, 1029-1044 (1998)

13. Rey, W.: Introduction to Robust and Quasi-Robust Statistical Methods. Springer, Berlin (1980)

14. Yang, G.Z., Burger, P., Kilner, P.J., Karwatowski, S.P., Firmin, D.N.: Dynamic Range Extension of Cine Velocity Measurements Using Motion Registered Spatio-Temporal Phase Unwrapping. Journal of Magnetic Resonance Imaging 6, 495-502 (1996)

15. Cerqueira, M.D., Weissman, N.J., Dilsizian, V., Jacobs, A.K., Kaul, S., Laskey, W.K., Pennell, D.J., Rumberger, J.A., Ryan, T., Verani, M.S.: Standardized myocardial segmentation and nomenclature for tomographic imaging of the heart: a statement for healthcare professionals from the Cardiac Imaging Committee of the Council on Clinical Cardiology of the American Heart Association. Circulation 105, 539-542 (2002) 